\title{
Variabilidad de la precipitación en la ciudad de Punta Arenas, Chile, desde principios del siglo XX
}

\author{
Rainfall variability at Punta Arenas City, Chile, \\ since beginning of 20th century
}

Álvaro González-Reyes ${ }^{1}$, Juan Carlos Aravena ${ }^{2}$, Ariel A. Muñoz ${ }^{3}$,
Pamela Soto-Rogel ${ }^{2}$, Isabella Aguilera-Betti ${ }^{3}$ \& Isadora Toledo-Guerrero

\section{Resumen}

Registros de precipitación instrumental extensos y continuos son escasos en América del Sur, y aún más en altas latitudes. Estos registros son útiles para contrastar reconstrucciones climáticas del pasado y calibrar modelos climáticos regionales. Pocas ciudades de Chile (e.g. Santiago, Concepción, Valdivia, Punta Arenas), poseen observaciones meteorológicas extensas desde mediados del siglo XIX o principios del siglo XX. El análisis de estos registros es también fundamental para evaluar los cambios recientes en el clima respecto de períodos previos, información especialmente útil para delinear políticas de adaptación. Este trabajo analizó la pluviometría de Punta Arenas y tuvo como objetivos: i) evaluar los cambios mensuales, estacionales y anuales de la precipitación en los períodos 1900-2014 y 1990-2014, ii) evaluar la recurrencia de eventos de sequías, y iii) analizar la relación entre la precipitación y el Modo Anular del Sur (SAM). Los resultados evidenciaron una fuerte variabilidad decadal a multi-decadal en la precipitación anual y en cada estación del año. Reducciones significativas se observaron en la precipitación anual durante el período 19002014, siendo más acentuadas entre 19902014. En este último período, la precipitación de primavera y verano presentó una reducción significativa, sugiriendo una extensión de las condiciones estivales. En invierno, un significativo incremento fue observado desde el año 1990. La recurrencia de eventos extremos de sequías severas también se incrementó posterior al año
1990. Relaciones negativas y significativas entre la actividad SAM y la precipitación anual se observaron en marzo y en el intervalo septiembrediciembre. La reducción en la precipitación de la ciudad de Punta Arenas estaría estrechamente relacionada con la actividad SAM, el cual modularía la variabilidad de las precipitaciones a escalas temporales interanuales $e$ interdecadales.

\section{Palabras clave:}

Modo Anular del Sur SAM, precipitación, Punta Arenas.
Abstract
Extended and continuous instrumental records of precipitation are scarce in South America, even more at high latitudes. These records are useful to validate past climatic reconstructions and calibrate regional climatic models. Few Chilean cities (e.g. Santiago, Concepción, and Valdivia) present long meteorological observations, since mid-19th century and/or the beginning of 20th century. The analysis of these records is also key to assessing recent changes In the climate with

\footnotetext{
1 Instituto de Ciencias de la Tierra, Facultad de Ciencias. Universidad Austral de Chile, Valdivia, Chile.

$\Sigma$ gonzalezreyesalvaro@gmail.com versidad de Magallanes, Punta Arenas, Chile
}
2 Dirección de Programas Antárticos y Subantárticos, Uni-
3 Laboratorio de Dendrocronología y Estudios Ambientales, Instituto de Geografía, Pontificia Universidad Católica de Valparaíso (PUCV), Valparaíso, Chile.


respect to previous periods. This Information is especially useful for delineating adaptation policies. This paper studied the Punta Arenas rainfall record seeking for the following aims: i) to evaluate monthly, seasonal, and annual variations of rainfall records over the 19002014 and 1990-2014 interval, ii) to evaluate the recurrence of drought extreme events in annual precipitation, and iii) to analyze the relationship between precipitation and the Southern Annular Mode (SAM). The findings evidenced strong decadal to multi-decadal variability in the annual and seasonal precipitation. A significant decrease of the annual precipitation was observed across the 1900-2014 period, becoming more severe in the 1990-2014 period. In this last period, spring and summer precipitation shows also a significant negative trend, suggesting an extension of summer conditions. In winter season, a significant increase was observed since 1990. Extreme drought events have increased since the 1990 year as well. Significant and negative relationships were observed between SAM activity and annual precipitation in March, and September to December months. Decreasing rainfall pattern in Punta Arenas is closely associated with the SAM variability at inter-annual to inter-decadal time scales.

\section{Key words:}

Southern Annular Mode SAM, rainfall records, Punta Arenas.

\section{INTRODUCCIÓN}

Registros meteorológicos instrumentales de precipitación extensos y continuos son escasos en América del Sur (AS), sin embargo, excepcionalmente existen mediciones desde mediados del siglo XIX y principios del siglo XX. Dentro de AS, algunas ciudades con registros cercanos al año 1850 en Brasil son: Fortaleza (1849) y Río de Janeiro (1851). En Argentina, la ciudad de Buenos Aires comenzó su registro de las precipitaciones en el año 1861. En el caso de Chile, las mediciones se iniciaron en el año 1866 en Santiago, mientras que en Concepción los registros comenzaron en 1876. El año 1860 comienzan los registros en Puerto Montt, y el año 1853 en la ciudad de Valdivia gracias al trabajo de Carlos Anwandter, constituyendo el registro más antiguo de precipitación en Chile. Estos registros extensos tienen gran utilidad, por ejemplo, para evaluar históricamente eventos de El Niño Oscilación del Sur (ENSO), como el ocurrido entre los años 1877-1878, el cual generó consecuencias a nivel global (Aceituno et al. 2009); evaluar cambios en el régimen de precipitaciones históricas desde el año 1853 en la ciudad de Valdivia (González-Reyes \& Muñoz, 2013); y evaluar la recurrencia de eventos de sequías en la ciudad de Santiago de Chile, desde el año 1867 (González-Reyes, 2016). En adición, estos extensos registros han permitido calibrar y generar reconstrucciones climáticas de las precipitaciones para los últimos 1000 años en Chile Central (Le Quesne et al. 2006).

En ciudades emplazadas a altas latitudes de AS, como es el caso de la ciudad de Punta Arenas, existe una larga tradición de mediciones de registros meteorológicos, los cuales también comenzaron en el siglo XIX. En sus inicios esta tradición se basó en la iniciativa de los establecimientos educacionales salesianos que se fundaron en la ciudad hacia finales de dicho siglo. De este modo, el $1^{\circ}$ de diciembre de 1887 se fundó el Observatorio Meteorológico "Monseñor Fagnano" en el Liceo San José de Punta Arenas, ubicado en el centro de la ciudad, que bajo el cuidado de sus profesoresmisioneros ha funcionado ininterrumpidamente hasta la actualidad (Santana, 1984; Santana et al. 2009). A partir de 1930, la Fuerza Aérea de Chile efectuó mediciones meteorológicas en sus instalaciones de Bahía Catalina, a $4 \mathrm{~km}$ del centro de la ciudad; luego, en el año 1964, esta estación fue trasladada al aeropuerto Carlos Ibáñez del Campo, $24 \mathrm{~km}$ al norte de la ciudad, lugar donde funciona actualmente bajo la administración de la Dirección Meteorológica de Chile (DMC). Una tercera estación meteorológica aún en funcionamiento en la ciudad de Punta Arenas es la establecida en el Instituto de la Patagonia en el año 1970 con el nombre de "Jorge C. Schythe" (Santana et al. 2009). Otras instituciones, como la Armada de Chile y la Dirección General de Aguas (DGA), también mantienen en la actualidad registros meteorológicos, lo que configura una 
interesante red de estaciones para distintos estudios espacio-temporales de la precipitación en Punta Arenas. Los registros más extensos dentro de esta red, son clave para poder analizar los cambios de la precipitación a múltiples escalas temporales, desde años hasta periodos decadales y multi-decadales.

Estudios previos sobre la precipitación en la región se han enfocado en determinar patrones espacio temporales a lo largo de Patagonia, y su relación con forzantes climáticos de escala global como ENSO y el Modo Anular del Sur (SAM) u Oscilación Antártica (AAO) (Thompson \& Wallace, 2000; Schneider \& Gies, 2004; Aravena \& Luckman, 2009). En adición, investigaciones hechas por Aravena y Luckman (2009) muestran que la precipitación en Patagonia está mayormente influenciada por la actividad SAM, que por la actividad de El Niño Oscilación del Sur (ENSO). El Modo Anular del Sur o SAM es un patrón de variabilidad climática extra-tropical, con signos de anomalías opuestas entre latitudes altas (e.g ubicación de la ciudad de Punta Arenas) y latitudes medias (e.g ubicación de la ciudad de Valdivia), el cual representa el primer componente principal de los campos de presiones al sur de los $20^{\circ} \mathrm{S}$ (Thompson \& Wallace, 2000). Este índice presenta una fase positiva y otra negativa, las cuales tienen efectos en el movimiento norte-sur del cinturón de vientos del oeste y la ruta de tormentas. Durante la fase positiva, SAM presenta anomalías de presión atmosférica positivas en latitudes medias y anomalías de presión negativas en latitudes altas, lo que resulta en una intensificación del anticiclón del Pacífico sur-oriental, y una disminución de la precipitación en latitudes medias. Por el contrario, durante la fase negativa de SAM, se presentan anomalías negativas de presión en latitudes medias y anomalías positivas en latitudes altas, con una intensificación del ciclón de baja presión en latitudes altas, y un incremento de la precipitación en latitudes medias (Thompson \& Wallace, 2000). El cinturón de vientos del oeste presenta una contracción hacia Antártica y como consecuencia, los sistemas de altas presiones están desplazados hacia la zona austral del hemisferio sur.

En los últimos 50 años, la actividad SAM ha presentado una significativa tendencia a la fase positiva, particularmente en el verano austral (Thompson \& Wallace, 2000; Marshall, 2003). Esto se ha expresado en anomalías positivas de temperatura en el extremo sur de AS y la Península Antártica (Thompson \& Solomon, 2002; Gillett et al. 2006; Garreaud et al. 2009, Garreaud et al. 2013). Si bien, estudios previos han evaluado cambios temporales en la precipitación a escala regional (e.g. Aravena \& Luckman, 2009), una evaluación de las tendencias de la precipitación de Punta Arenas a escala anual y estacional a lo largo de todo el siglo XX aún no ha sido llevada a cabo. Por otro lado, si bien se conoce que la actividad SAM influencia las precipitaciones de Patagonia, aún se desconoce los meses donde esta relación ejerce mayor influencia sobre la precipitación de Punta Arenas, y también la escala temporal a la cual ocurre dicha relación (e.g. años, décadas, multi-décadas). En este sentido, el presente trabajo tuvo como objetivos: I) Evaluar las tendencias de la precipitación histórica anual y estacional en la ciudad de Punta Arenas entre los años 1900-2014, con énfasis en el periodo 1990-2014. ii) Evaluar la recurrencia de eventos extremos de sequías, y iii) Evaluar la relación entre la precipitación y la actividad del Modo Anular del Sur (SAM).

\section{MÉTODOS}

El área de estudio se emplaza en la ciudad de Punta Arenas (5309'45” S; 7054'29” O), área geográfica cuyo tipo de clima predominante es el estepárico frío (Luebert \& Pliscoff, 2008). La precipitación en la región está concentrada particularmente en las estaciones de otoño y verano, la cual cae en forma líquida, mientras que en invierno cae en forma sólida. La media anual acumulada entre los años 1900-2000 corresponde a 416 milímetros.

Los análisis de este trabajo se realizaron utilizando el registro pluviométrico de la estación Punta Arenas compilado y actualmente manejado por la Dirección Meteorológica de Chile (DMC). Este registro cubre el período 1900-2014. Cabe mencionar que la serie de precipitación utilizada en este trabajo se ha compuesto por las mediciones tomadas en sus inicios por la estación 
Fagnano, la que se ha juntado posteriormente con el registro de la DMC. No obstante, esta serie temporal compuesta presenta un patrón de variabilidad temporal coherente con el registro de otras estaciones de precipitación en Patagonia (e.g. Río Gallegos), si se comparan dentro del período 1931-2014. Los cambios temporales de la precipitación fueron evaluados a nivel anual y estacional. La precipitación anual fue considerada como la precipitación acumulada entre enero a diciembre. Estacionalmente, la precipitación de verano se definió desde diciembre del año previo hasta febrero del año actual (DEF), el Otoño desde marzo a mayo (MAM), el Invierno de junio a agosto (JJA) y la primavera de septiembre a noviembre (SON). En todas las estaciones del año fue considerada la sumatoria de las precipitaciones en dicha combinación de meses.

Los cambios en la precipitación han sido evaluados en dos intervalos temporales: 19002014, correspondiente a todo el registro, y en el período 1990-2014. Las tendencias se evaluaron mediante la prueba de MannKendall (MK; Kendall, 1975) y a través de regresiones lineales simples (RL). La prueba estadística de MK es no paramétrica, robusta y ampliamente usada para evaluar tendencias en series temporales ambientales (Hess et al. 2001). Para su evaluación se utilizó el paquete estadístico "Kendall" (McLeod, 2015), el cual fue computado en la plataforma R - Project (R Core Team, 2016). Este mismo programa fue usado para computar las RL. En adición, se utilizaron análisis estadísticos complementarios en base a la prueba de Mann-Whitney para identificar cambios temporales significantes en el promedio de las precipitaciones a través del tiempo (ver paquete "Changepoint" para R; Killick, 2016). Adicionalmente, se utilizaron filtros cúbicos Spline de 10 años en orden de resaltar posibles variaciones decadales en la precipitación de Punta Arenas. El análisis espectral MTM fue computado con el objetivo de identificar los principales ciclos dentro de la precipitación anual de Punta Arenas.

La evaluación de la recurrencia de eventos extremos de sequías se determinó a través de una función de estimación de densidad de Kernel tras establecer valores umbrales de sequía extraídos desde la propia serie de precipitación anual acumulada, calculados entre el período 19002014. Se consideró un evento de sequía extrema como valores de precipitación menor o igual a los percentiles 5 y 10 (P05=249, $1 \mathrm{~mm}$; P10=295,2

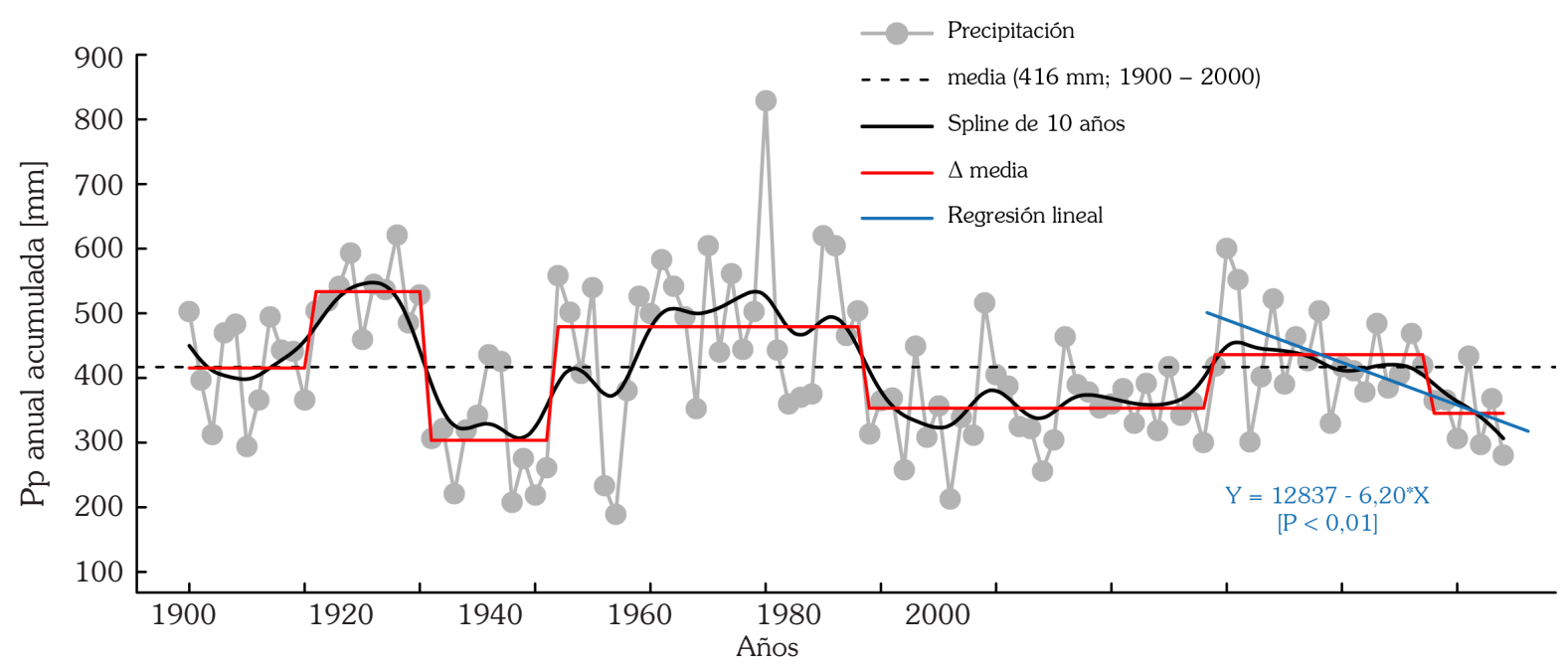

Fig. 1. Precipitación anual acumulada de la ciudad de Punta Arenas entre los años 1900 - 2014.

Las líneas segmentadas representan el promedio calculado para el siglo XX (416 milimetros). La línea negra continua representa un filtro Spline de 10 años en orden de resaltar la variabilidad decadal que contiene la serie de precipitación. La línea roja continua representa los cambios temporales significativos en la media $(\Delta)$ de las precipitaciones en base a la prueba de "Mann-Whitney". Los cambios temporales son estadísticamente significativos con un 95\% de confianza. La ecuación en base a un modelo de regresión lineal (RL) fue calculada en base al período 1990 - 2014. 
$\mathrm{mm})$. Eventos de sequía severa fueron dados por el percentil 15 y el percentil 20 (P15= 306,5 $\mathrm{mm}$; P20 $=319,9 \mathrm{~mm})$, y de sequía moderada considerando el percentil 25 (P25 $=334,8$ $\mathrm{mm})$. El ancho de banda utilizado para hacer la estimación de la recurrencia de sequías fue de 25 años, y los intervalos de confianza a un $95 \%$ de significancia fueron estimados en 1000 simulaciones utilizando bootstrap (Cowling et al. 1996).

Para evaluar la relación entre la precipitación anual de Punta Arenas y la actividad SAM se calcularon correlaciones $r$ de Pearson con los índices SAM a nivel mensual. Dado que existe una variedad de índices del Modo Anular del Sur, en este trabajo se utilizaron los índices SAM NCAR-NCEP y el índice SAM calculado por Visbeck (2009), basado en las mediciones instrumentales de la presión atmosférica a nivel del mar a lo largo del hemisferio Sur, las que cubren el período 1887-2011. En adición y de manera complementaria, se computó un análisis Wavelet Cruzado y de Coherencia Espectral. Ambos análisis tuvieron como objetivo evaluar la existencia de posibles similitudes espectrales entre la serie temporal de precipitación de Punta Arenas y la serie temporal del Índice SAM. Cabe mencionar que estudios previos desarrollados entre la precipitación instrumental en Patagonia y su relación con el modo SAM no han indagado en las relaciones dentro de un dominio tiempo - frecuencia entre ambas variables. Este punto podría proveer nueva información respecto a la dinámica temporal entre SAM y la precipitación en altas latitudes, como la ciudad de Punta Arenas.

\section{RESULTADOS}

La precipitación anual acumulada de la ciudad de Punta Arenas registró una serie de períodos húmedos y secos a lo largo del siglo XX y la primera década del siglo XXI (Fig. 1). Al considerar el promedio de la precipitación anual acumulada para el último siglo $(416 \mathrm{~mm}$; período 1900-2000), fue posible observar intervalos temporales de mayor precipitación entre los años: 1910-1920, 1930-1960 y entre 1990 -2008. Contrariamente, períodos secos o de

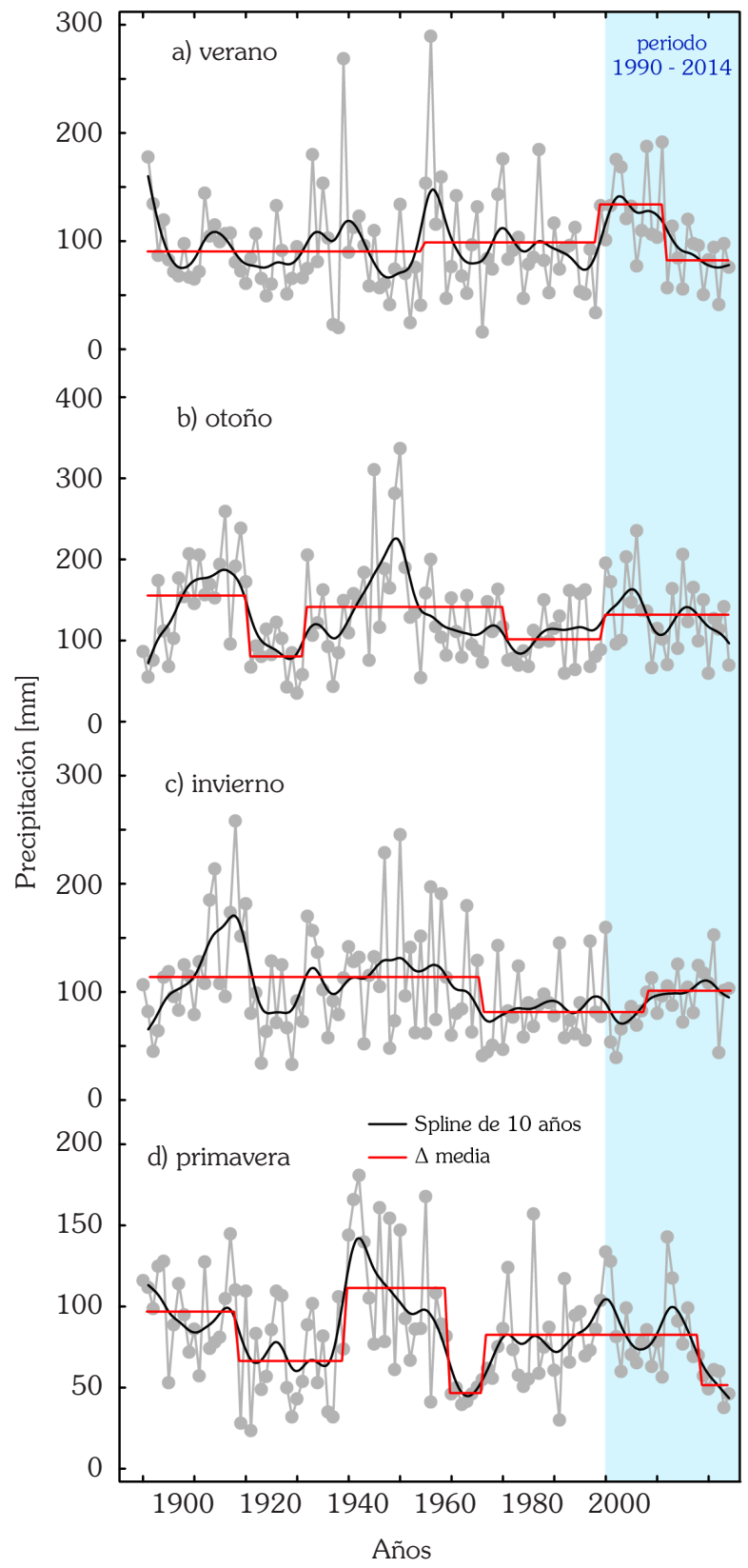

Fig. 2. Precipitación estacional en a) verano, b) otoño, c) invierno y d) primavera. Líneas de color negro representan un filtro cúbico Spline de 10 años utilizado para resaltar la variabilidad decadal e interdecadal en las series de precipitación. Líneas de color rojo representan los cambios temporales significativos en la media en base a la prueba de "Mann-Whitney". Los cambios temporales fueron estadísticamente significativos con un $95 \%$ de confianza. 


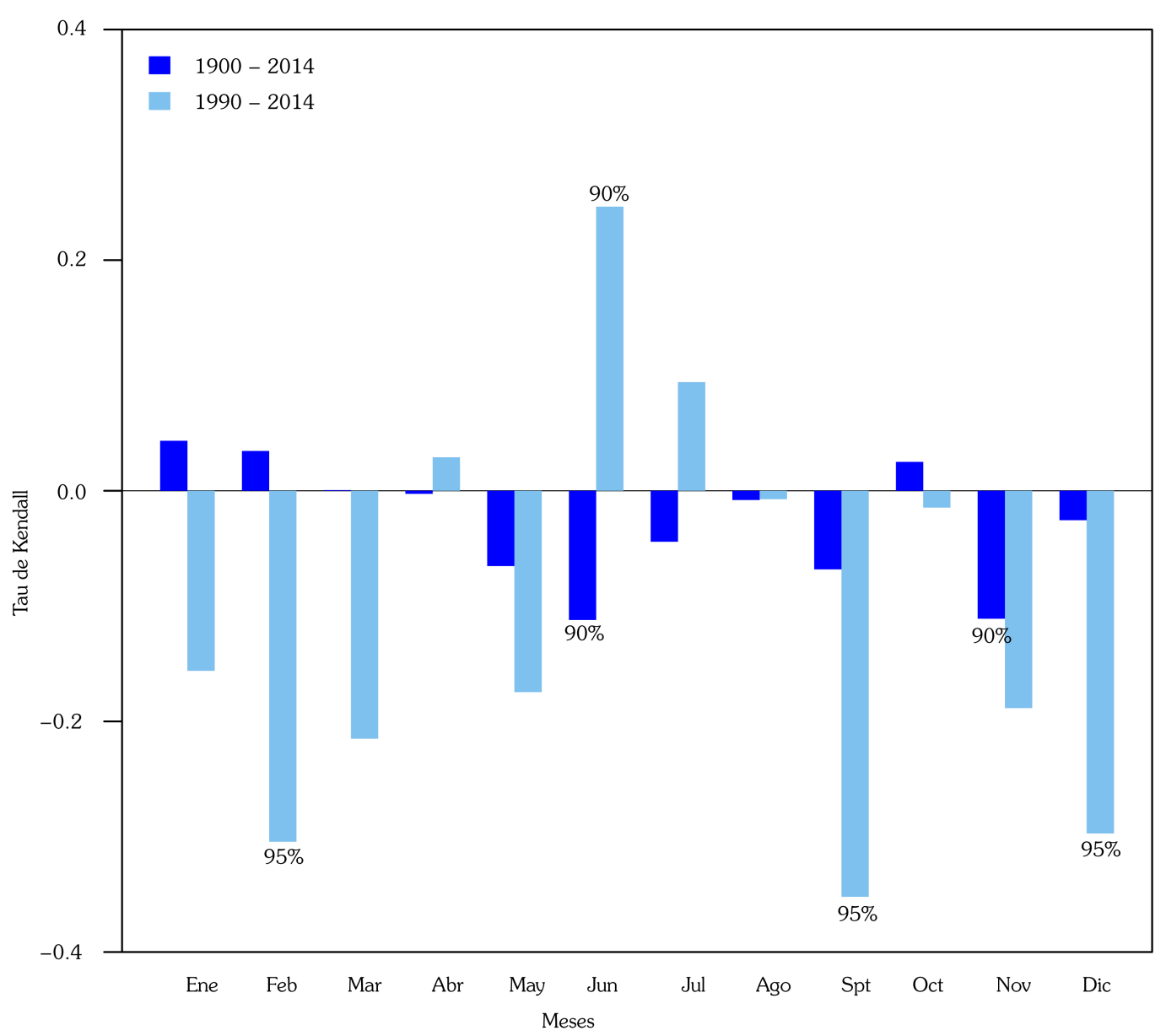

Fig. 3. Resumen de las tendencias mensuales de la precipitación de Punta Arenas en base a la prueba de Mann-Kendall, y calculado entre los dos períodos analizados: 1900 - 2014 y 1990 - 2014. El nivel de significancia estadística se muestra en los casos en que estas tendencias alcanzaron niveles significativos.

menor precipitación fueron registrados entre los años: 1919-1933 y 1961-1989. Estos períodos húmedos y secos que ocurren en escala decadal y multi-decadal se identifican con mayor claridad al aplicar una suavización a la serie de precipitación anual utilizando un filtro Spline de 10 años, y también un análisis temporal de cambios en la media, donde se observan siete intervalos con variaciones estadísticamente significativas (Fig. 1). Estos cambios temporales en la media ocurrieron en periodos de entre 7 y 28 años, aproximadamente.

La precipitación anual acumulada presentó una tendencia negativa marginalmente significativa en base a la prueba MK. si se considera el período 1900-2014. Sin embargo, esta tendencia fue más marcada y estadísticamente significativa si se considera el intervalo 1990-2014 (RL y MK; Fig. 1). La precipitación por estaciones del año, al igual que la precipitación anual acumulada, mostró una serie de períodos húmedos y secos a lo largo del siglo XX y principios del XXI (Fig. 2). Un intervalo húmedo fue registrado entre los años 1940-1960, el cual es observado en la mayoría de las estaciones del año, a excepción de la estación de verano (Fig. 2a). Un período seco se observó posterior al año 1960, con una longitud variable de acuerdo a la estación del año (Figs. 2b, c, d). Posterior a este año, en estaciones como otoño, el aumento de la precipitación fue paulatino (Fig. 2b), mientras que en invierno $y$ especialmente en primavera el aumento de la precipitación fue más marcado al menos hasta 1990. 
Por su parte, a partir del año 1990, la precipitación de verano y primavera han registrado una negativa y significativa tendencia (MK; Figs. $2 \mathrm{a}$ y 2d). Contrariamente, en la precipitación invernal una tendencia positiva, incrementándose significativamente desde el año 1990 (MK; Fig. 2c). La precipitación de la estación de otoño no una clara y significativa tendencia en los períodos analizados (Fig. 2b).

La precipitación de Punta Arenas a escala mensual registró tendencias positivas y negativas a lo largo del período 1900-2014. No obstante, tendencias marginalmente significativas (MK) se obtuvieron solo en los meses de junio y noviembre (Fig. 3). En ambos meses, estas tendencias fueron negativas. Un mayor número de meses, en comparación al período 1900-2014, registraron cambios significativos en su tendencia dentro del intervalo 1990-2014. Los meses donde la precipitación mostró cambios significativos y una tendencia negativa fueron: febrero, septiembre y diciembre (MK), mientras que una tendencia positiva y marginalmente significativa se obtuvo para el mes de junio (MK).

\section{Eventos extremos de sequías}

Los eventos extremos de sequías basados en la precipitación anual acumulada mostraron una fuerte variación durante el siglo XX $\mathrm{y}$ principios del siglo XXI. La recurrencia de eventos de sequía moderada $(\mathrm{P} 25)$ registraron un incremento desde año 2000, mientras que eventos de sequía severa (P15 y P20) presentaron un incremento desde el año 1990. Una condición más seca a la registrada en las últimas décadas se observó entre los años 1910-1940, con una mayor recurrencia de sequías alrededor del año 1930 (cada 2,9 años aproximadamente para el P25). En este período, todos los umbrales de sequías presentaron una mayor recurrencia (Fig. 4). Otro período de mayor recurrencia de sequías moderadas a severas fue observado entre los años 1960-1980, con una recurrencia de aproximadamente 2,9 años para el P25 alrededor del año 1970.

\section{Ciclos de la precipitación de Punta Arenas}

Utilizando herramientas de análisis espectral como el análisis MTM se observó que la serie de precipitación anual acumulada registra ciclos de variabilidad estadísticamente significativos de: 2,1, 2,5, 7,1 y 28,5 años (Fig. 5a). Dentro de estos ciclos, se destaca el ciclo de 7,1 años de periodicidad, el cual tiene una fuerte y significativa señal entre los años 1920-1960

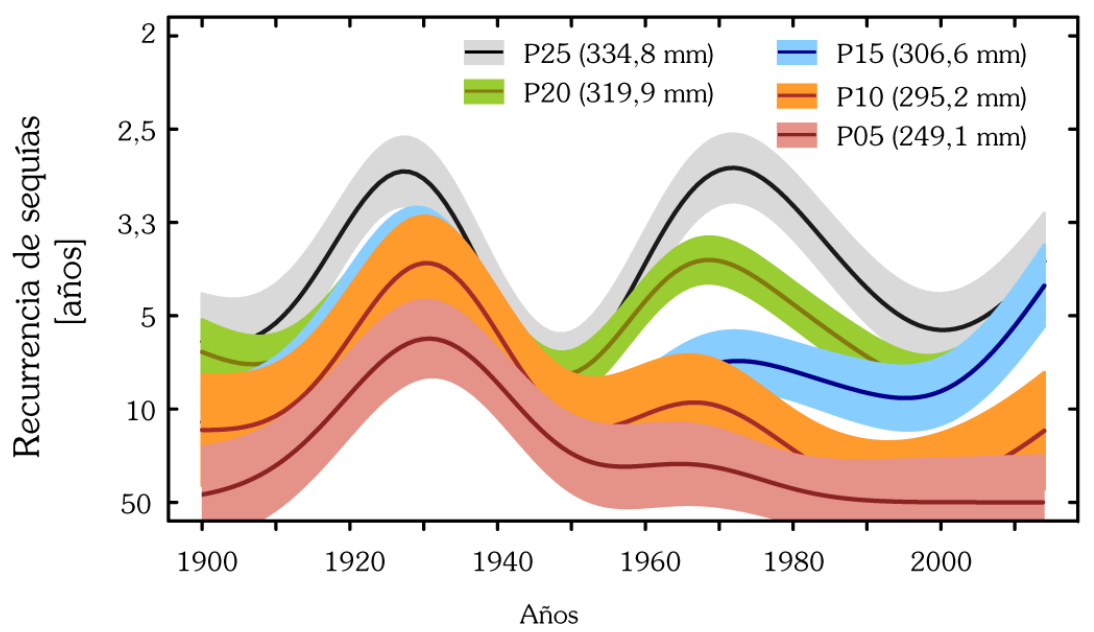

Fig. 4. Recurrencia de eventos de sequías (líneas enteras), definidas como: sequías moderadas (P25), severas (P15 y P20) y extremas (P05 y P10). El computo se ha basado en la precipitación anual acumulada de Punta Arenas entre el período 1900 - 2014. El ancho de banda utilizado para estimar la recurrencia de eventos extremos fue de 25 años. En cada una de estimaciones, la banda de color representa los límites de confianza calculados al $95 \%$ usando la técnica bootstrap obtenida en base a 1000 simulaciones. 


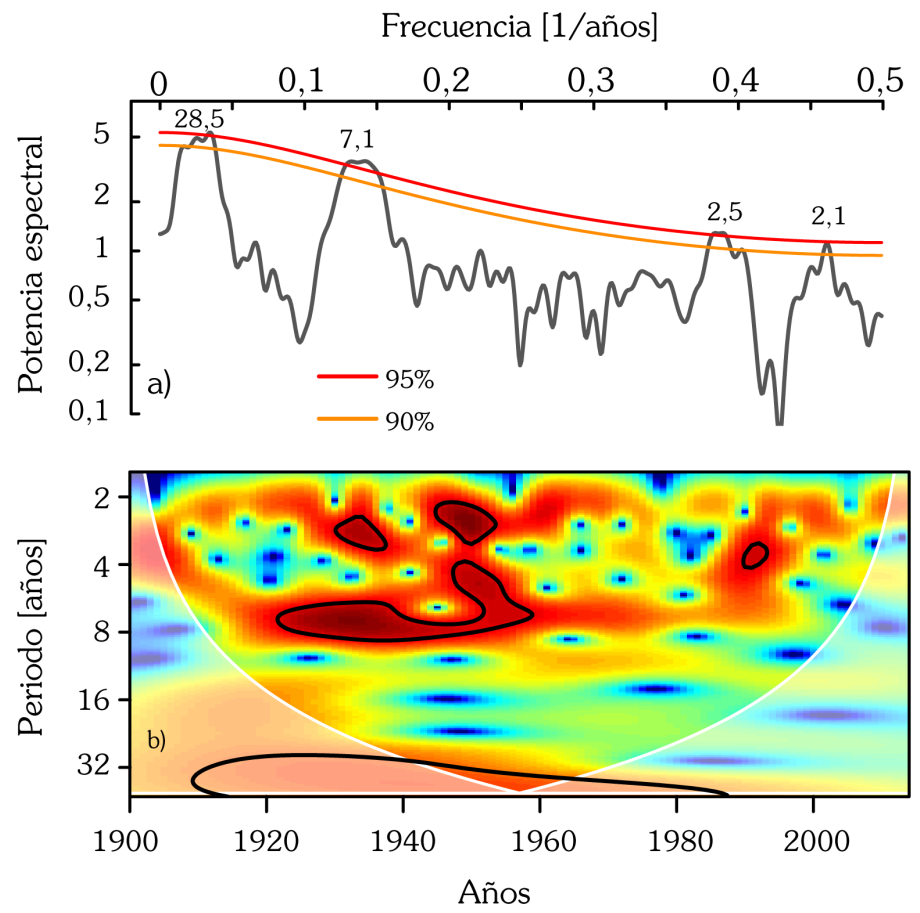

Fig. 5. a) Análisis MTM computado para la precipitación anual de Punta Arenas entre los años 1900 - 2014. Los ciclos significantes a diferentes valores de probabilidad son mostrados sobre el espectro. b) Análisis Wavelet de la serie de precipitación anual. Señales de colores rojo intenso se refieren a una mayor potencia espectral. Las áreas enmarcadas en líneas de color negro representan señales espectrales estadísticamente significantes a un $95 \%$ de confianza. El cono de influencia (línea blanca) marca el límite donde el análisis Wavelet es confiable.

observada a través del análisis Wavelet (95\%; Fig. $5 b)$. Este ciclo de 7,1 años además retiene un $23 \%$ de la variabilidad total de la precipitación. El ciclo mayor de 28,5 años ha sido también obtenido mediante el análisis Wavelet, el cual muestra una potente y significativa señal espectral entre los años 1910-1990; sin embargo, dicha señal espectral se encuentra mayormente fuera del cono de influencia, lo cual aumenta la incertidumbre de este resultado (Fig. 5b).

\section{Relación entre el Modo Anular del Sur SAM y la precipitación de Punta Arenas}

A escala mensual, la precipitación anual de Punta Arenas registró una correlación estadísticamente significativa con la actividad de SAM NCAR-NCEP en los meses de: marzo $(r=-$ $0,28)$, octubre $(r=-0.36)$, noviembre $(r=-0.22)$ y diciembre $(r=-0.25)$. Dentro de estos meses, noviembre presentó una correlación marginalmente significativa. Estos resultados fueron obtenidos en base a correlaciones calculadas en el período 1948-2011 y con el indice SAM sin su tendencia lineal. En una escala estacional, la primavera fue la única estación del año que obtuvo una correlación estadísticamente significativa con el índice SAM NCAR-NCEP $(r=-0.30)$.

En un contexto temporal de largo plazo, al contrastar las señales espectrales de la precipitación anual y el índice anual de SAM de Visbeck (2009) entre losaños 1900-2011, se obtuvo una coherencia espectral estadísticamente significativa en ciclos de variabilidad de: 2,3, 2,6, 7,4 y 15,4 años (Fig. 6a). Los ciclos de alta frecuencia (2,3 y 2,6 años), y de media frecuencia (7,4 años), mostraron una fuerte y común potencia espectral entre los años 19301960, y en el período 1990-2014 (Fig. 6a). En adición, una potente señal espectral combinada de ciclos de alta y media frecuencia (2-7 años aprox.) fue observada desde el año 1990 al presente (Fig. 6b). Una intensa y significativa señal alrededor de 

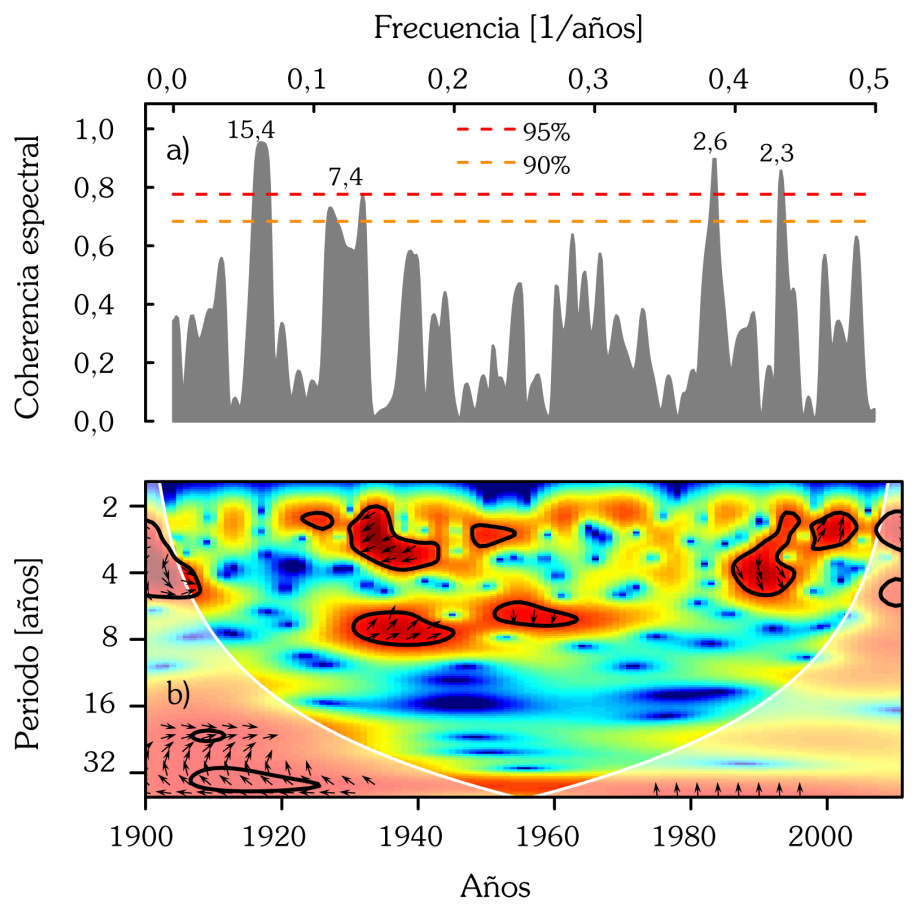

Fig. 6. a) Coherencia espectral entre el índice SAM anual basado en Visbeck (2009) con una remoción de la tendencia lineal y la precipitación anual de Punta Arenas. El intervalo temporal de comparación fue 1900 - 2011. Los ciclos significantes a diferentes valores de probabilidad son mostrados sobre el espectro. b) Análisis Cross - Wavelet entre el SAM anual y precipitación anual que resume las señales espectrales de alta potencia comunes entre ambas variables. Las áreas enmarcadas en líneas de color negro representan señales espectrales estadísticamente significantes a un $95 \%$ de confianza. El cono de influencia (línea blanca) marca el límite donde el análisis Wavelet es confiable. Las flechas representan un estado de fase (dirección hacia la derecha) o anti-fase (dirección hacia la izquierda) entre la precipitación y el SAM.

los 7 años también fue registrada en los períodos $1930-1945$ y $1955-1965$.

\section{DISCUSIÓN}

\section{Tendencias de la precipitación anual, estacional y mensual}

El extenso registro instrumental de la precipitación de la ciudad de Punta Arenas ha permitido evaluar las tendencias a nivel mensual, estacional y anual, durante el período 19002014, con énfasis en el período 1990-2014. Un análisis previo de la precipitación de la ciudad, para un intervalo de 96 años (1888-1984) fue realizado por Santana (1984). Este autor utilizó treintenios (e.g. 1901-1930; 1931-1960) como períodos fijos para sus análisis. En el período en que se sobreponen los análisis de Santana
(1984) con los de este trabajo, se distinguen similitudes en los intervalos húmedos y secos de la precipitación. No obstante, el uso de treintenios en una serie que presenta una fuerte componente temporal de variación decadal a interdecadal, como es el caso de la precipitación de Punta Arenas, implica mezclar tendencias a la hora de evaluarlas. En este sentido, el método de treintenios impide reconocer cambios temporales en la tendencia e intervalos de más o menos precipitaciones, como los resultados reportados en este trabajo. A una escala anual, una reducción de las precipitaciones fue observada desde comienzos del siglo XX, siendo más marcada desde el año 1990 hacia el presente en algunas estaciones del año. Por ejemplo, las estaciones de verano y primavera registraron reducciones significativas. La reducción de las precipitaciones en verano estaría estrechamente relacionada con 
un flujo zonal circumpolar anómalo (Garrreaud et al. 2013). Por su parte, el invierno fue la única estación que mostró una tendencia positiva desde 1990, con incrementos significativos en la precipitación de junio. Este incremento significativo registrado en la precipitación de invierno podría estar relacionado con un incremento en las temperaturas mínimas que registra la ciudad de Punta Arenas (Aravena et al. 2002). Lo anterior, se manifestaría en una mayor precipitación en forma líquida caída sobre la ciudad. Este aumento observado en la precipitación de invierno podría estar vinculado a anomalías en los vientos Oestes a baja altura en la zona del Pacífico sudeste (Garreaud et al. 2013).

La negativa tendencia en las precipitaciones observada en el registro instrumental de más de 100 años de la ciudad de Punta Arenas, y que es expuesta en este trabajo, es también concordante con resultados obtenidos por otros autores, los cuales han evaluado cambios en la precipitación en otras partes de la Patagonia mediante el uso de modelos generales de circulación (GCM). Estos GCM reportan una disminución de las precipitaciones en Patagonia concentrada alrededor de la banda de los $45^{\circ} \mathrm{S}$ (Watterson, 2000; Cai \& Watterson, 2002; Karoly, 2003; Gupta \& England, 2006). Por otro lado, el análisis de la precipitación de Punta Arenas permite su comparación con otros registros extensos de precipitación de Chile, desde la zona central hasta la Patagonia. Por ejemplo, en la ciudad de Valdivia $\left(39,8^{\circ} \mathrm{S}\right)$, dentro de un clima Templado lluvioso, se observó una negativa tendencia en las precipitaciones desde mediados del siglo XIX (González-Reyes \& Muñoz, 2013). En esta ciudad la estación de otoño ha registrado una mayor reducción en sus precipitaciones desde el año 1901. En una escala mensual, la precipitación de abril y septiembre registraron reducciones significativas (González-Reyes \& Muñoz, 2013). Lo anterior es distinto de lo observado en Punta Arenas, en donde las reducciones son moderadamente significativas en primavera y en los meses de junio y noviembre, considerando el período 1900-2014 como referencia. A pesar de estas diferencias, tanto para Valdivia como para Punta Arenas, reducciones de precipitación en las estaciones de otoño y primavera respectivamente, expresan una extensión de las condiciones estivales y por tanto un aumento de la probabilidad de sequías. En la zona central de Chile, en el caso de la ciudad de Santiago $\left(33,4^{\circ} \mathrm{S}\right)$, las sequías extremas se incrementaron a partir del año 1950 (González-Reyes, 2016), al igual que en la ciudad de Valdivia (González-Reyes \& Muñoz, 2013), lo cual no ocurrió claramente hasta después de 1990 en Punta Arenas. Este resultado refuerza las diferencias en el patrón de precipitaciones descrito para la zona central bajo un clima tipo Mediterráneo, la zona centro-sur bajo un clima Templado-lluvioso, y la zona austral o patagónica bajo un clima del tipo EstepáricoFrío. La disminución de las precipitaciones en el análisis de la ciudad de Santiago ocurrió especialmente en los meses de mayo, junio y julio. Estos meses son relevantes en esta zona, ya que las precipitaciones se concentran en mayor medida en los meses de invierno dentro de un clima tipo Mediterráneo (González-Reyes, 2016). No obstante, estas observaciones son opuestas a las obtenidas para las variaciones de la precipitación en Punta Arenas, en donde el invierno presentó un incremento significativo de las precipitaciones en las últimas décadas (desde 1990 en adelante).

\section{Recurrencia de eventos extremos de sequías}

La recurrencia de eventos extremos de sequías en la ciudad de Punta Arenas fue mayor alrededor de 1930 y 1970 , y con un incremento desde el año 1990 en adelante (ver Fig. 4). Parece ser que los cambios en eventos extremos después de 1990 en conjunto con la extensión de las condiciones estivales, podrían estar afectando la dinámica de los ecosistemas boscosos en Patagonia. Durante las últimas décadas, grandes extensiones de bosques del genero Nothofagus afectados por pérdida de vigor y mortalidad, han motivado una serie de trabajos dirigidos a evaluar los efectos de la variabilidad climática sobre la regeneración y la mortalidad episódica de especies arbóreas nativas en la Patagonia (Villalba \& Veblen, 1998; Suarez \& Kitzberger, 2008; Paritsis \& Veblen, 2011, Rodríguez-Catón et al. 2016). En la actualidad 
los bosques de la Patagonia forman parte de uno de los ecosistemas mayormente afectados por la variabilidad del clima a nivel global (Allen et al. 2010). Registros desde el año 1850 han documentado mortalidad de bosques de Nothofagus pumilio (Poepp. \& Endl.) Krasser provocada por ataques de insectos (Ormiscodes), que estarían favorecidos por eventos cálidos en el clima (Paritsis \& Veblen, 2011). Estos eventos podrian ser más frecuentes bajo la extensión de las condiciones estivales en la zona, según lo observado en la precipitación de Punta Arenas. Considerando el aumento de sequías en Punta Arenas, es necesaria una evaluación en otros componentes de los ecosistemas asociados a la ciudad, y en los servicios que estos proveen a la sociedad para ser utilizada en políticas de desarrollo y adaptación al cambio climático en Punta Arenas y sus alrededores.

Componentes cíclicos de la precipitación de Punta Arenas

Una característica de la precipitación de Punta Arenas es su marcada variabilidad decadal a multi-decadal, la que es observada durante todo el siglo XX y principios del siglo XXI (ver Figs. 1, 2 y 3). Estas fluctuaciones decadales $y$ multi-decadales en la precipitación ocurrirían con una frecuencia de entre 7 a 28 años, según los análisis espectrales (ver Fig. 5). El ciclo de baja frecuencia cercano a 28 años podría explicar los cambios significativos en la media identificados en la precipitación anual entre los años 1931-1959 y 1960-1989 (ver Fig. 1). En estos intervalos temporales además se han registrado los mayores incrementos en la recurrencia de sequías moderadas, severas y extremas (período 19311959), y sequías moderadas a severas (período 1960-1989; ver Fig. 4). La variación decadal a multi-decadal de la precipitación de Punta Arenas exhibe períodos prolongados de baja pluviosidad, lo cual podría ser interesante de analizar en futuras investigaciones en un contexto de variabilidad en el clima de Patagonia. Dicha variación temporal en las precipitaciones podría traducirse periodos de mayor y menor vulnerabilidad ante eventos de sequías en la ciudad de Punta Arenas. Este resultado podría ser relevante para el manejo de los recursos hídricos en la región, y también la generación de pronósticos relacionados con el abastecimiento y disponibilidad de agua futura para la ciudad.

Relaciones entre la precipitación de Punta Arenas y el forzante climático SAM

La variabilidad temporal de la precipitación de Punta Arenas está relacionada con la actividad SAM a múltiples escalas temporales, la cual también varía a lo largo del año. La actividad de SAM de primavera (septiembre a noviembre), registró además correlaciones negativas significantes a escala mensual con la precipitación en dichos meses, siendo la única estación que obtuvo correlaciones significantes. Este resultado señala que la actividad SAM ejerce mayormente una modulación sobre las precipitaciones de primavera en Punta Arenas. En este sentido, la modulación producida por SAM sobre el régimen de precipitaciones podría explicar los significativos cambios registrados en primavera. No obstante, la actividad del modo SAM estaría ejerciendo una modulación de las precipitaciones a otras escalas temporales más allá de la variabilidad estacional e interanual (e.g. decadal interdecadal). A pesar de esto, existe una fuerte variación temporal de alta frecuencia entre ambas series temporales, las que registraron una potente señal espectral común de 2,6 años de periodicidad; sin embargo, este ciclo es intermitente $y$ ha mostrado una potente señal espectral reforzada desde 1990 al presente (Fig. 6b). Otro resultado a destacar es el coherente ciclo común de variabilidad cercano a 15 años entre el modo SAM y la precipitación anual (ver Fig. 6a); no obstante, este no fue identificado a través del análisis Wavelet cruzado. Nosotros creemos en base a la Fig. 6b, que es necesario hacer un tratamiento espectral (e.g. filtro de pasa bajo), en orden de eliminar la señal espectral de alta frecuencia y estudiar este ciclo de baja frecuencia con una mayor profundidad, dado que podría estar generando fluctuaciones en la precipitación, como se observó mediante los cambios temporales en la media a lo largo de todo el intervalo 1900-2014 (ver Fig. 1).

El ciclo significativo de 7 años obtenido en la serie temporal de precipitación anual, y que a 
su vez registró tener una coherencia espectral significativa con el modo SAM, parece ser un ciclo importante en la variabilidad de la precipitación de Punta Arenas. Este ciclo es capaz de explicar aproximadamente el $23 \%$ de la varianza total de la precipitación anual. Este ciclo común obtenido en base a los análisis espectrales de coherencia y wavelet cruzado demostrarían que la actividad SAM modula el régimen de precipitación anual de Punta Arenas a dicha escala temporal. A parte de este ciclo de 7 años, existe también una modulación de la actividad SAM sobre las precipitaciones, pero a una escala interanual. Este hecho queda demostrado en la Fig. 6b, donde se observa una intermitente y significativa señal espectral dentro de la banda de periodicidad de entre 2-4 años, si se compara la precipitación anual y el modo SAM a lo largo de todo el período analizado. Estudios futuros relacionados con la variabilidad de las precipitaciones y su interacción con el modo SAM en AS, podrían focalizarse en evaluar la existencia de los ciclos reportados en este trabajo en otros registros instrumentales extensos de ciudades ubicadas a altas latitudes (e.g. Puerto Williams, Ushuaia, Río Gallegos). Este punto podría ser relevante a fin de comprender si la modulación ejercida por el modo SAM sobre el régimen de precipitaciones a lo largo de otras partes de la Patagonia actúa de manera similar a lo obtenido para la precipitación anual de la ciudad de Punta Arenas.

Los resultados expuestos en el presente trabajo exhiben la modulación provocada por la actividad SAM sobre las precipitaciones de la ciudad de Punta Arenas, especialmente en primavera $y$ principios del verano. Esta modulación, que se produciría debido a que SAM (una medida del gradiente de presión atmosférica entre latitudes medias y altas) genera cambios en la ruta de tormentas sobre el hemisferio Sur, Este modo de variabilidad del clima en medias y altas latitudes, ha presentado una mayor intensificación y una tendencia positiva desde el año 1958 (Marshall, 2003). En adición, está intensificación del gradiente estaría generando un desplazamiento de la ruta de las tormentas y núcleo de los vientos Oestes hacía las altas latitudes (Toggweiler, 2009). Cabe mencionar que la intensificación que se ha registrado en el
SAM desde la segunda mitad del siglo XX tiene una fuerte componente antrópica, ya que dichos cambios estarían relacionados con variaciones en la concentración de gases de efecto invernadero y con cambios en el ozono estratosférico (Toggweiler, 2009; Thompson et al. 2011). Los cambios registrados en la precipitación de Punta Arenas desde la segunda mitad del siglo XX estarían en parte influenciados por la actividad humana.

\section{CONCLUSIONES}

La precipitación anual acumulada en la ciudad de Punta Arenas registró una negativa y significativa tendencia desde el año 1900. Esta reducción demostró ser más acentuada en el período 1990-2014. No obstante, la precipitación mostró una fuerte fluctuación temporal a una escala decadal a multi-decadal. Esta característica fue observada a lo largo de todo el siglo XX y principios del siglo XXI. A escala estacional. la precipitación acumulada de primavera y verano presentó un significativo patrón de reducción, particularmente entre los años 1990-2014. Contrariamente, la precipitación de invierno es la única estación del año donde se ha incrementado significativamente la precipitación desde el año 1990 al presente.

Un incremento en la recurrencia de eventos extremos de sequías severas ha sido observado desde el año 1990 al presente en la precipitación anual. No obstante, condiciones de mayor aridez a las registradas en décadas recientes se observaron en la precipitación de Punta Arenas entre los años 1910-1940.

La precipitación anual de Punta Arenas y el Modo Anular del Sur exhiben relaciones a múltiples escalas temporales (interanual a interdecadal). Una fuerte modulación producida por la actividad de SAM sobre el régimen de precipitaciones estaría ocurriendo mediante ciclos de 7 años y a través de otros de mayor longitud. La precipitación de primavera fue la que registró la más clara influencia de la actividad SAM. Estas reducciones estarían incrementando el período seco y la probabilidad de ocurrencia de sequías en Punta Arenas. El estudio y la comprensión del efecto de la variabilidad del clima sobre los sistemas socio-económicos y los 
ecosistemas patagónicos, surge como un desafío para el desarrollo de políticas de adaptación en una de las ciudades más australes del mundo.

\section{AGRADECIMIENTOS}

Álvaro González-Reyes agradece a la Corporación Nacional de Ciencia y Tecnología CONICYT por su apoyo doctoral (CONICYTPCHA/Doctorado Nacional/2016-21160642). Juan Carlos Aravena y Pamela Soto-Rogel agradecen el financiamiento del proyecto FONDECYT $\mathrm{N}^{\circ} 1130381$. Ariel A. Muñoz, Isabella Aguilera-Betti e Isadora Toledo-Guerrero agradecen al Centro del Clima y la Resiliencia (CR)2 (FONDAP 15110009), y al proyecto FONDECYT No11161061. Los autores agradecen también a dos revisores anónimos que ayudaron a mejorar este manuscrito.

\section{LITERATURA CITADA}

Aceituno, P., Prieto, M. del R., Solari, M.E., Martínez, A., Poveda, G., \& Falvey, M. (2009). The 1877-1878 El Niño episode: associated impact in South America. Climatic Change, 92, 389-416.

Allen, C. D., Macalady, A. K., Chenchouni, H., Bachelet, D., McDowell, N., Vennetier, M.,...\& Gonzalez, P. (2010). A global overview of drought and heat-induced tree mortality reveals emerging climate change risks for forests. Forest ecology and management, 259(4), 660-684.

Aravena, J. C., Lara, A., Wolodarsky-Franke, A., Villalba, R., \& Cuq, E. (2002). Treering growth patterns and temperature reconstruction from Nothofagus pumilio (Fagaceae) forests in the upper tree line of Southern Chilean Patagonia. Revista Chilena de Historia Natural, 75, 361-376. Aravena, J. C., \& Luckman, B. H. (2009). Spatiotemporal rainfall patterns in Southern South America. International Journal of Climatology, 29, 2106-2120.

Cai, W., \& Watterson, I. G. (2002). Modes of Interanual Variability of the Southern Hemisphere Circulation Simulated by the CSIRO Climate Model. Journal of Climate, 15, 1159- 1174.
Cowling, A., Hall, P., \& Phillips, M. J. (1996). Bootstrap confidence regions for the intensity of a Poisson point process. Journal of the American Statistical Association, 91, 1516-1524.

Garreaud, R., Vuille, M., Compagnucci, R., \& Marengo, J. (2009). Present-day South America Climate. Palaeogeography, Palaeoclimatology, Palaeoecology, 281, 180-195.

Garreaud, R., Lopez, P., Minvielle, M., \& Rojas, M. (2013). Large-Scale Control on the Patagonian Climate. Journal of Climate, 26, 215-230.

Gillet, N. P., Kell, T. D., \& Jones P. D. (2006). Regional climate impacts of the Southern Annular Mode. Geophysical Research Letters, 33, doi: 10.1029/2006GL027721

González-Reyes, A., \& Muñoz, A. A. (2013). Cambios en la precipitación de la ciudad de Valdivia (Chile) durante los últimos 150 años. Bosque, 34(2), 191-200.

González-Reyes, A. (2016). Recurrencia de eventos de sequías en la ciudad de Santiago de Chile desde mediados del siglo XIX. Revista de Geografía Norte Grande, 64, 21-32.

Gupta, A. S., \& England, M. H. (2006). Coupled ocean-atmosphere-ice response to variations in the Southern Annular Mode. Journal of Climate, 19, 4457-448

Hess, A., Hari, I., \& Malm, W. (2001). Linear trend analysis: a comparison of methods. Atmospheric Environment 35, 52115222.

Karoly, D. J. (2003). Ozone and climate change. Science, 302, 236-237.

Kendall, M. G. (1975). Rank Correlation Methods (4a Edición). London UK: Editorial Charles Griffin.

Killick, R. (2016). Methods for Changepoint Detection. Disponible en: https://github. com/rkillick/changepoint/

Le Quesne, C., Stahle, D. W., Cleaveland, M. K., Therrell, M. D., Aravena, J. C., \& Barichivich, J. (2006). Ancient Austrocedrus tree-ring chronologies used to reconstruct Central Chile precipitation variability from A.D. 1200 to 2000. Journal of Climate, $19,5731-5744$. 
Luebert, F., \& Pliscoff, P. (2008). Sinopsis bioclimática y vegetacional de Chile. (1 $1^{a}$ Edición.) Santiago, Chile: Editorial Universitaria.

Marshall, G. (2003). Trends in the Southern Annular Mode from Observations and Reanalyses. Journal of Climate, 16, 41344143.

McLeod, A. I. (2015). Kendall rank correlation and Mann-Kendall trend test. Disponible en https://github.com/cran/Kendall/

Paritsis, J., \& Veblen, T. T. (2011). Dendroecological analysis of defoliator outbreaks on Nothofagus pumilio and their relation to climate variability in the Patagonian Andes. Global Change Biology, 17(1), 239-253.

R Core Team (2016). R: A language and environment for statistical computing. R Foundation for Statistical Computing, Vienna, Austria. Disponible en www.Rproject.org

Rodríguez-Catón, M., Villalba, R., Morales, M., \& Srur, A. (2016). Influence of droughts on Nothofagus pumilio forest decline across northern Patagonia, Argentina. Ecosphere. doi: $10.1002 /$ ecs2.1390

Santana, A. (1984). Variación de las precipitaciones de 97 años en Punta Arenas como índice de posibles cambios climáticos. Anales del Instituto de la Patagonia, 15(1), 50-60.

Santana, A., Butorovic, N., \& Olave, C. (2009). Variación de la temperatura en Punta Arenas (Chile) en los últimos 120 años. Anales del Instituto de la Patagonia, 37(1), 85-96.

Schneider, C., \& Gies, D. (2004). Effect of El
Niño-Southern Oscillation on southernmost South America precipitation at $53^{\circ} \mathrm{S}$ revealed from NCEP-NCAR reanalyses and weather station data. International Journal of Climatology, 24, 1057-1076.

Suarez, M. L., \& Kitzberger, T. (2008). Recruitment patterns following a severe drought: Longterm compositional shifts in Patagonian forests. Canadian Journal of Forest Research. 38, 3002-3010.

Thompson, D., \& Wallace, J. (2000). Annular Modes in the Extratropical Circulation. Part I: Month-to-Month Variability. Journal of Climate, 13(694), 1000-1016.

Thompson, D., \&Solomon, S. (2002). Interpretation of Recent Southern Hemisphere Climate Change. Science, 296, 895-899.

Thompson, D., Solomon, S., Kushner, P., England, M., Grise, K. M., \& Karoly, D. (2011). Signatures of the Antarctic ozone hole in Southern Hemisphere surface climate change. Nature Geoscience, 4, 741-769.

Toggweiller, J. (2009). Shifting Westerlies. Nature Geoscience, 323, 1435.

Villalba R., \& Veblen, T. (1998). Influences of large-scale climatic variability on episodic tree mortality at the forest-steppe ecotone in northern Patagonia. Ecology, 79, 26242640.

Visbeck, M. (2009). A station-based Southern Annular Mode index from 1884 to 2005. Journal of Climate, 22, 940-950.

Watterson, I. G. (2000). Southern midlatitude zonal wind vacillation and its interaction with the ocean in GCM simulations. Journal of Climate, 13, 562-578. 